\title{
RESET
}

Recherches en sciences sociales sur Internet

\section{Les « civic tech » à l'épreuve des partis politiques}

Les plateformes participatives de l'Union pour un Mouvement Populaire (UMP) et du Parti Socialiste (PS)

"Civic tech» with the test of the political parties. Participatory Platforms of the

Union for Popular Movement (UMP) and the Socialist Party (PS)

\section{Anaïs Theviot et Éric Treille}

\section{(2) OpenEdition}

\section{Journals}

Édition électronique

URL : http://journals.openedition.org/reset/906

DOI : 10.4000/reset.906

ISSN : 2264-6221

Éditeur

Association Recherches en sciences sociales sur Internet

Référence électronique

Anaiis Theviot et Éric Treille, "Les " civic tech » à l'épreuve des partis politiques », RESET [En ligne],

7 | 2018, mis en ligne le 29 octobre 2018, consulté le 19 avril 2019. URL : http://

journals.openedition.org/reset/906 ; DOI : 10.4000/reset.906

Ce document a été généré automatiquement le 19 avril 2019.

(c) Association Recherches en sciences sociales sur Internet 


\title{
Les « civic tech » à l'épreuve des partis politiques
}

\author{
Les plateformes participatives de l'Union pour un Mouvement Populaire \\ (UMP) et du Parti Socialiste (PS) \\ "Civic tech» with the test of the political parties. Participatory Platforms of the \\ Union for Popular Movement (UMP) and the Socialist Party (PS)
}

Anaïs Theviot et Éric Treille

\section{Introduction}

1 En l'espace de dix ans, l'Internet s'est propulsé sur le devant de la scène politique française. Ce qui était encore marginal au début des années 2000 est devenu la nouvelle norme du combat électoral. Aucune campagne ne peut désormais se concevoir sans recourir aux outils numériques, au risque de se demander si les «fact checkeurs » n'ont pas remplacé les distributeurs de tracts, les joutes de tweets les batailles de colleurs d'affiches.

2 Cet état des lieux est à la fois vrai et faux. Il est en effet difficile de nier l'essor rapide de ces nouvelles techniques de mobilisation. La campagne présidentielle de 2017 en a ainsi offert de nombreuses illustrations, à travers notamment l'usage de la plateforme NationBuilder ${ }^{1}$ utilisée aussi bien par Jean-Luc Mélenchon que par Alain Juppé (Theviot 2016a). En même temps, de nombreux angles morts continuent à subsister sur la réalité de l'ouverture des partis politiques au numérique et sur l'effet de démocratisation des outils du web pour rompre avec les pratiques traditionnelles. L'intégration progressive du numérique dans les stratégies de communication par les partis français doit nous inviter à questionner le fonctionnement même des organisations politiques (Chadwick, 2007 ; Stromer-Galley, 2014; Theviot, 2018). Le développement de l'internet partisan et notamment des plateformes participatives internes aux formations est-il ainsi l'annonciateur de nouvelles formes d'actions politiques moins hiérarchisées, organisées en réseaux, plus ponctuelles et temporaires (Wright, 2012a et 2012b) ? Ou s'agit-il, plus 
prosaïquement, d'un nouvel exemple de « routines innovantes» (Gourgues, 2012)? Dit autrement : ces nouvelles expériences de participation ne constituent-elles pas un nouvel avatar de la " démocratie d'élevage ", selon la formule de Laurent Mermet, en opposition aux stratégies de «démocratie sauvage» constituées en dehors des dispositifs institutionnels (Mermet, 2007), à l'image de la mise en œuvre d'autres pratiques partisanes non conventionnelles comme les "primaires ouvertes ou citoyennes" (Lefebvre \& Treille, 2016)?

3 À la suite de travaux déjà menés sur le débat public (Monnoyeur-Smith, 2011; Badouard \& Mabi, 2015), nous souhaitons montrer que ce découpage est aussi malmené par les formations politiques. En effet, la démocratie dite numérique, censée être plus interactive, s'est imposée en réponse aux crises traversées par la démocratie électorale représentative (Blondiaux, 2011; Rosanvallon, 2015). De nouvelles pratiques citoyennes se sont alors développées, axées sur l'empowerment des citoyens (Bacqué \& Biewener, 2013) ou le community organizing (Talpin, 2016), intégrant souvent le numérique comme moyen de reconfigurer les pratiques politiques (Coleman, 2005 ; Breindl \& Francq, 2008; Cardon, 2010). La multiplication de mouvements politiques tels que Ma voix qui parle «d'hacker » l'Assemblée nationale ou la mise en place de primaires citoyennes en ligne avec par exemple lesprimaires.org a permis aux chercheurs de s'interroger sur ce que le numérique fait au renouvellement des modes de participation politique (Collin, 2015). Pour unifier ces différentes démarches, s'est alors imposé le concept de « civic tech » dans le discours des acteurs. Apparu au début des années 2010 dans un contexte mêlant défiance citoyenne et appropriation croissante des outils numériques, ce mot valise a fait depuis l'objet de multiples définitions pour rassembler tout type d'initiatives politiques cherchant à transformer et à améliorer la démocratie via des dispositifs numériques.

4 À la recherche de nouveaux équipements participatifs et d'instruments inédits d'inclusion militante, les partis politiques se sont également emparés du vocable des « civic tech » pour pallier aux critiques sur leur manque de prise en compte de la parole citoyenne et s'«ouvrir» plus largement aux non-adhérents. A une nuance près cependant: ces «civic tech" à l'épreuve des partis s'intègrent parfaitement dans l'institution partisane et diffèrent ainsi des technologies à visées citoyennes, qui se développent soit en faveur de l'engagement civique (Lefébure \& Sécail, 2016), soit en s'inscrivant dans des logiques de contre-démocratie ou de contre-pouvoir (Rosanvallon, 2015). En cela, elles s'apparentent davantage à la typologie mise en place par le Secrétariat d'État au Numérique en 2016, des «technologies pour la démocratie », c'est-àdire des initiatives œuvrant dans le champ institutionnel.

$5 \quad$ Les cas des deux plateformes participatives mises en place par l'UMP - Les Créateurs des possibles - et par le PS - La Coopol- traduisent bien ce double mouvement entre volonté de remettre le citoyen au cœur du jeu politique grâce aux technologies numériques et encadrement de la parole citoyenne sur des dispositifs partisans. Ces plateformes participatives créées à quelques jours d'intervalle en 2010, dans un même mouvement d'émulation technique et politique, ont ainsi donné une forme matérielle à des discours de rénovation des partis, en intégrant les discours émergents sur les «civic tech». Ces expérimentations ont-elles eu des effets réellement « disruptifs » dans les façons de faire de la politique ou s'agit-il d'un nouvel avatar du «travail de normalisation » (Vedel \& Ward, 2006) effectué par les formations politiques, voire de «blanchiment démocratique » éloigné du modèle initial de transformation de la vie publique (Mabi, 2017) ? 
Dans ce cadre, l'ambition de cet article est d'analyser ces deux exemples de domestication partisane de l'ouverture au numérique. Elle est aussi de souligner les nombreuses tensions qui traversent la rencontre des « civic tech » et des partis politiques, partagés entre le souhait de faire participer davantage les citoyens à la vie politique via le numérique et une réalité technologique dominée par une offre limitée d'interactivité et une maximisation des barrières à l'entrée. Face aux revendications des militants, ces outils, très encadrés dans un premier temps par l'institution partisane, ont dans un second temps été portés par les usagers (et non le parti) en laissant aux participants la gestion des débats. Mais malgré les ambitions affichées, les partis politiques étudiés ne se détachent pas de leur mode d'organisation et de leur souhait de contrôler leur communication. Pis, les développements les plus récents du numérique partisan soulignent un usage encore plus restrictif des pratiques collaboratives offertes par le web. Si le numérique s'est imposé comme une ressource politique désormais majeure, il semble qu'il le soit davantage comme un élément de la «nouvelle science électorale " (Pène, 2013) inspiré du modèle américain que comme un accélérateur de la participation en ligne.

7 Pour nous aider à dépasser les discours sur la rénovation des partis politiques, nous nous appuyons sur une cinquantaine d'entretiens semi-directifs avec les créateurs, gestionnaires et usagers partisans et non partisans de ces plateformes.

Les plateformes participatives en ligne, mises en place par l'UMP et le PS en 2010, se sont inspirées de l'émergence du mouvement des « civic tech » aux États-Unis, en cherchant pour Les Créateurs du possible à faire remonter les idées citoyennes aux partis et pour La Coopol à " ouvrir » les débats partisans aux non-adhérents. Toutefois, ces ambitions se sont vues fortement contrariées par le manque de participation en ligne des citoyens et par la captation de ces offres militantes par des personnes déjà membres de ces organisations, soulignant en cela le travail d'appropriation suscité et généré par ces plateformes créées pour et par des institutions partisanes traditionnelles.

\section{Les Créateurs des possibles ou une plateforme partisane sans communauté militante}

Encore marginal au début des années 2000, le numérique s'est en effet imposé sur le devant de la scène politique française lors de la campagne présidentielle de 2007. La « campagne participative » menée par Ségolène Royal et son association Désir d'avenir a été pour beaucoup dans ce changement profond de la nature de la communication politique. Fortement inspirée du succès de mybarackobama.com, la représentante socialiste avait su alors gagner la bataille de la modernité politique en s'appuyant sur des interfaces numérisées d'implications militantes et de débats en ligne (Desquinabo, 2008). Les formes de l'engagement à un parti s'en trouvaient également fortement assouplies puisque les frontières physiques de l'organisation n'étaient plus un obstacle au militantisme, même s'il était «assis ». Cette élection a enfin obligé les partis à investir à leur tour le web politique et à traduire, en dehors des périodes de campagne, la nouvelle conception de militance - plus ouverte et plus participative - qui avait alors émergé.

La transformation de sites internet de candidats en plateformes participatives de partis ne va cependant pas de soi. L'UMP en a ainsi fait l'expérience en 2010. En lançant le 7 janvier 2010, le site communautaire - Les Créateurs de possibles-, Xavier Bertrand, le 
Secrétaire général de l'UMP, a montré qu'il n'était pas si simple pour une formation politique de "hacker» la démocratie partisane et de transposer dans une organisation, même sous une forme institutionnalisée, des modalités d'implication citoyenne éloignées des cultures partisanes. Présenté comme "une nouvelle manière de passer à l'action grâce à Internet : vous n'êtes plus spectateur mais véritablement acteur du débat citoyen $\|^{2}$, le réseau social de l'UMP a été avant tout un échec pour ses initiateurs.

En effet, si cette proposition technique et politique a rappelé celle de la plateforme participative de Désirs d'avenir - certains militants socialistes avaient même fortement critiqué cette imitation « qui se faisait passer pour une innovation $»^{3}-$, la similitude ne fut que technique. Le réseau de l'UMP ne rencontra pas le même écho que le site de campagne de Ségolène Royal. Il a ainsi rapidement éprouvé des difficultés pour trouver son public puis a fermé, après moins d'un an d'existence.

Le site de l'UMP s'est ainsi heurté à un paradoxe. Construit pour donner la parole aux citoyens, il n'a pas réussi à concerner le premier public du parti : ses adhérents. Faute de participants non encartés, notamment hors période de campagne électorale, la plateforme annoncée comme collaborative est restée inerte car sans membre actif et donc sans échange : «ce site n'est pas un 'réseau', puisque les individus en sont absents $»^{4}$.

Une vision tronquée du web et plus largement de la spécificité des « civic tech » peut être évoquée pour expliquer ce mauvais ciblage du public. En ligne, les discussions politiques ont souvent lieu là où on ne les attend pas. De même, les plateformes dédiées sont avant tout destinées aux adhérents ou à des publics fortement politisés et non aux citoyens "lambda ». Pour attirer des participants, il est donc important de cibler, à l'image des stratégies marketing développées par Barack Obama en 2008, des types particuliers d'intervenants aux débats. Selon Jennifer Lees-Marshment (2012), cette technique permet ainsi de mieux repérer les besoins politiques et les aspirations de l'électorat, ce qui participe à l'élaboration par les partis politiques de discours adaptés aux attentes.

«L'UMP s'était dit: 'On va créer un réseau social comme Facebook'. Pourquoi créer quelque chose qui existe déjà ? Première erreur. Et deuxième erreur : on n'a pas su dire ce que c'était. Donc on a dit : 'C'est un réseau créé par l'UMP, mais qui n'est pas pour les militants UMP. C'est pour tout le monde'. Donc en gros, ceux qui ne sont pas UMP disent: 'C'est créé par l'UMP, donc je n'y vois pas l'intérêt'. Et ceux qui sont UMP se disent: 'Oui enfin bon, en même temps, c'est pas un outil pour nous mais pour tout le monde. À quoi ça va nous servir ?'. »"

De fait, s'adresser en priorité aux citoyens «lambda " peut paraître incongru pour une plateforme mise en place par un parti politique hors période de campagne électorale et témoigne d'une mauvaise compréhension des stratégies à adopter en ligne.

"L'efficacité d'Internet ne réside pas dans sa capacité à atteindre les 'électeurs médians', non politisés, ce qui est toujours la doxa de la plupart des consultants politiques. Si on conserve cette perspective, alors Internet n'est qu'un outil mineur, un complément par rapport aux outils traditionnels de campagne. (...) Sa singularité en revanche est qu'il permet de mobiliser les citoyens les plus engagés en générant des bénévoles et des financements (...). La Toile n'est donc pas un moyen de communiquer avec le public en général, mais un moyen de communiquer entre leaders et activistes. » (Vergniolle de Chantal, 2011)

15 Pour que le citoyen puisse avoir connaissance de son existence et s'y rendre, sa médiatisation doit être maximale. Or, au sein même de l'UMP, certains acteurs ont souligné des erreurs « originelles » sur la conception de l'outil et sur l'élaboration du plan 
de communication. Celui-ci, selon Pierre Chassat, directeur de la communication de l'UMP, a été centré sur l'esthétique du dispositif et non sur son utilité.

«Donc mauvais ciblage, mauvaise conception et en plus, on a fait une mauvaise communication, c'est-à-dire qu'on a vendu un outil, on a vendu... Mais comme le précédent site est sorti. On a fait: 'Regardez, on a un bel outil'. On n'a pas communiqué sur les résultats, sur ce que ça allait permettre de faire mais seulement sur l'outil : 'Regardez, c'est beau'. Donc c'est à tout point de vue mauvais. Donc on a arrêté. " $^{6}$

Au-delà du plan de communication média, c'est aux adhérents (et notamment aux Jeunes Populaires [JP] sous la présidence de Benjamin Lancar) que la promotion de l'outil sur la Toile a été confiée. Il s'est agi de faire connaître à leurs cercles proches ce dispositif pour qu'eux-mêmes en parlent à leur entourage et puissent, par un effet boule de neige, toucher un maximum de personnes.

«Des travaux récents au Royaume-Uni et en Belgique ont souligné la possibilité d'un effet de mobilisation indirecte ou en deux étapes: les sites de campagne mobilisent les activistes qui vont eux-mêmes mobiliser des sympathisants dans des réseaux hors ligne. » [Nous traduisons] (Gibson, 2012 :83)

Ce procédé a cependant rencontré des difficultés en raison de la faible communauté en ligne des cyber-activistes de droite. De fait, l'UMP n'a pas une culture forte du membership hors période de campagne (Haegel, 2007)7 . De même, ses adhérents actifs en ligne étaient trop peu nombreux pour promouvoir efficacement ce dispositif auprès des internautes. La structure de l'UMP, se rapprochant plus d'un parti d'électeurs que d'un parti de militants, n'intègre pas les mêmes stratégies de mobilisation en ligne (Lefebvre \& Treille, 2016).

« Je pense que l'UMP a beaucoup plus de membres que de militants. Et ça, je m'en étais rendu compte notamment pendant la campagne. À un moment donné, je m'étais amusé parce qu'il y avait beaucoup de joutes verbales ou beaucoup de controverses sur le nombre de militants dont on pouvait disposer chacun et notamment sur le nombre de mails. Alors tout le monde balançait des chiffres faux. Un jour le PS disait deux cents, alors l'UMP disait deux cent cinquante. Puis trois cents... Et à la fin, on était à... Je ne sais plus combien, entre cinq cent mille et un million. Évidemment aucun de ces chiffres n'était vrai. Ils sont tous aussi farfelus avec rarement les moyens de pouvoir vérifier. Mais ce qui est certain, c'est que moi je m'étais amusé, on avait fait des tests, en se disant ce n'est pas grave, on va prendre une grosse newsletter que l'UMP envoie ou sarkozy.fr qui dit :'allez-y, allez à tel endroit poster des commentaires désobligeants sur Ségolène Royal'. Et puis ben voilà, s'ils disent qu'ils l'ont envoyée à cinq cent mille personnes, logiquement, il doit y avoir quelques commentaires derrière. Quand vous en avez moins de quinze, c'est que soit vous n'en avez pas cinq cent mille et ce n'est évidemment pas le cas. Soit et c'est les deux en réalité, le peu de gens à qui vous l'envoyez ne sont pas très impliqués pour le faire. Et en fait, c'est les deux. Globalement ce n'est pas très glorieux. On se rend compte quand même qu'à la fin, la force militante que l'on est capable de faire bouger, de mobiliser, elle n'est pas énorme. Ça reste une petite minorité. $»^{8}$

18 En outre, sa position de parti au pouvoir ${ }^{9}$, au moment du lancement de ce réseau social, ne lui a pas permis de se construire une communauté en ligne. Les cyber-militants proUMP n'étaient pas organisés sur la Toile, contrairement aux blogueurs anti-Sarkozy. L'architecture de ce dispositif n'était pas non plus en adéquation avec ses ambitions. Contrairement à myBO ou Organizing for America ${ }^{10}$, Les Créateurs de possibles n'ont ainsi pas mis en scène ses membres: pas un visage, pas une photo de profil, pas un nombre d'amis, pas un pouce levé, pas un score associé à un nom. Enfin, cette plateforme n'a pas 
investi le champ de la «gamisation » afin d'attirer les internautes avec des sondages, des quizz, des scores, etc.

«Ils ont un président en exercice. Ça devrait être lui le créateur du possible. Ce n'est pas les autres. C'est ça le problème qu'ils ont eu. On dit aux gens des idées pour la présidence Sarkozy... Si Sarkozy n'avait pas été candidat, ils auraient fait les Créateurs du possible en 2005 peut-être oui, ça aurait peut-être marché mieux, mais là... Tout ça n'est pas déconnecté de la politique elle-même. Les Créateurs du possible... Leur candidat en exercice, c'est quand même difficile de demander aux gens : 'qu'est-ce que le président doit faire ?'. Personne n'a vraiment compris à quoi ça servait, y compris les gens à l'UMP, ça n'a jamais fonctionné. Après ils ont essayé de mettre en scène des trucs comme quoi Xavier Bertrand avait écouté des gens des Créateurs du possible pour telle suggestion qui était déjà dans le programme de Sarkozy quoi. Super quoi. Ils ont essayé de faire un coup de storytelling, mais les gens ne s'y sont pas trompés. C'est pour ça que ça n'a pas marché. »"11

La gêne a été tellement forte autour de l'échec de ce projet, au regard de l'investissement financier, que ses responsables ont alors préféré faire comme s'il n'avait jamais existé ${ }^{12}$. Pour preuve : à l'UMP, les stratèges du numérique qui en ont parlé le plus librement étaient ceux qui n'étaient pas dans l'équipe au moment de sa création ${ }^{13}$. Le résultat ne s'est pas fait attendre: né et porté sous le secrétariat général de Xavier Bertrand, le réseau a été fermé et l'équipe web renouvelée lorsque Jean-François Copé a pris la tête de l'UMP, ne permettant pas alors au parti de mesurer à froid l'ensemble des raisons techniques et politiques - qui ont expliqué la clôture de cette première initiative partisane de civic tech politique.

\section{La Coopol ou la domestication militante de l'ouverture partisane}

Cinq jours après le lancement du site communautaire de l'UMP, le PS a créé le 12 janvier 2010 son propre réseau social ${ }^{14}$, La Coopol, avec trois objectifs précis : «mieux organiser la vie de la section; interconnecter les militants pour favoriser les échanges et le débat interne; ouvrir le parti sur l'extérieur en permettant aux sympathisants de nous rejoindre $»^{15}$. Fort de son expérience de responsable du site de Désir d'avenir, Benoît Thieulin a mis en avant cette volonté d'ouverture: «La Coopol est une membrane qui permet de flouter la frontière entre sympathisants et militants ${ }^{16}$. La page d'accueil de la plateforme en témoigne : «Militants, sympathisants du PS ou de la gauche, chacun peut s'inscrire! $»^{17}$.

21 Encartés et non membres du parti ont donc été mis sur un même pied d'égalité (Barboni \& Treille, 2010). Tous deux représentent une force de mobilisation et d'action sur la Toile, le but du site étant de pouvoir sensibiliser un maximum d'internautes pour les échéances électorales de 2012. Pour le directeur du web du PS, ce nouveau réseau social représentait avant tout un réseau d'engagement politique à la fois ponctuel et temporaire :

«Il s'agit d'un outil pour numériser le PS, gérer le parti, discuter à travers toute la France. Ouvert aux sympathisants, qui se sentent souvent seuls, La Coopol sera aussi et surtout au service d'actions de terrain, ou de mobilisation collective comme la défense de la Poste dans laquelle s'était investie toute la gauche. Il doit permettre, sans prendre la carte, de s'engager. $»^{18}$

De fait, l'engagement en ligne propose une alternative à l'acte de prendre sa carte à un parti. Il ne s'agit plus d'inscrire nécessairement le lien militant dans la durée et 
l'attachement physique à un lieu de militantisme. La Coopol permet au contraire ne plus être a priori adhérent au PS pour agir en son sein. La rupture militante est ainsi importante puisque s'inscrire sur Internet en un ou deux clics à La Coopol ne requiert pas le même niveau d'engagement au sein du parti. En cela, l'outil numérique devait permettre de faire coexister au sein du PS des partis à "plusieurs vitesses » (Scarrow, 2015) donnant ainsi corps à l'injonction de « mise en responsabilité choisie » développée notamment pendant la campagne de Barack Obama en 2008.

La démarche mise en place était volontairement simple. Il suffisait de renseigner son nom, prénom, adresse mail et de certifier avoir pris connaissance de la charte de bonne conduite. Un mail était ensuite directement envoyé à l'adresse indiquée : "Merci d'avoir rejoint La Coopol, la coopérative politique du Parti socialiste. Tu peux désormais renseigner ton profil (...) $»^{19}$. L'emploi du tutoiement n'était pas neutre : ce changement de ton soulignait en cela l'appartenance nouvelle à un réseau de « coopains $»^{20}$. Pour les concepteurs du projet, l'objectif était de créer du lien entre les internautes, de faire naître un sentiment de proximité, voire d'amitié. La Coopol était, cette fois-ci, bien rattachée au parti puisqu'il n'y était pas fait mention de la gauche de façon globale, mais bien du PS.

Bien que le parti ait affiché une volonté d'ouverture du débat aux citoyens non encartés, ce sont pourtant en priorité les adhérents du PS qui ont participé en ligne. Ce confinement de la participation à des profils d'adhérents a amené à des concurrences entre motions, liés notamment au futur contexte des primaires, déjà visibles au sein des débats en ligne. Ainsi, dans les quatre premiers mois suivant le lancement de la plateforme, les discussions ont été rapidement très conflictuelles, notamment entre partisans de différents courants.

«Au début, on a eu pas mal de militants qui sont venus et qui se sont un peu écharpés. Tu es de telle motion... Donc je ne t'aime pas. Tu soutiens plutôt un tel, une telle, donc je ne t'aime pas. Ça pouvait parfois être assez violent. On a mis le bon ordre. Ça a pris environ trois ou quatre mois. (...) En réalité, c'étaient des partisans de la motion de Ségolène Royal qui souvent étaient aussi sur Désirs d'Avenir. Ils étaient déjà extrêmement organisés sur Internet de par la campagne de 2007: assez habiles, actifs sur les réseaux sociaux, sur Internet de manière générale. Quand on a ouvert la Coopol, ils sont venus assez rapidement, en masse. En l'occurrence, c'était quelque chose de plutôt positif. En revanche, les camarades qui soutenaient plutôt les autres motions, ont vu ça comme une invasion. 'La Coopol c'est celle de Ségolène Royal'. Il commençait à y avoir des débats où il y a eu des attaques qui étaient parfois vraiment au ras des pâquerettes. On a fait énormément de modération pendant trois ou quatre mois où on a expliqué d'une part qui on est, à quoi servait la Coopol. Le côté réunion était important. Le fait de dire on se met tous ensemble, on a des objectifs communs malgré nos différences. On peut bosser ensemble et discuter sans s'insulter. $»^{21}$

Dans les premiers mois du réseau, la modération effectuée par la Direction du web du PS à Solférino s'est avérée très stricte. Avec l'arrivée massive de partisans de Ségolène Royal sur La Coopol, les autres motions se sont senties vite exclues ou mal représentées et ont donc protesté. Pour éviter qu'elles ne fassent les gros titres des journaux ${ }^{22}$, ces luttes internes entre courants ont dû alors être cachées par le modérateur. L'objectif était de donner l'image d'un parti uni et dynamique. 


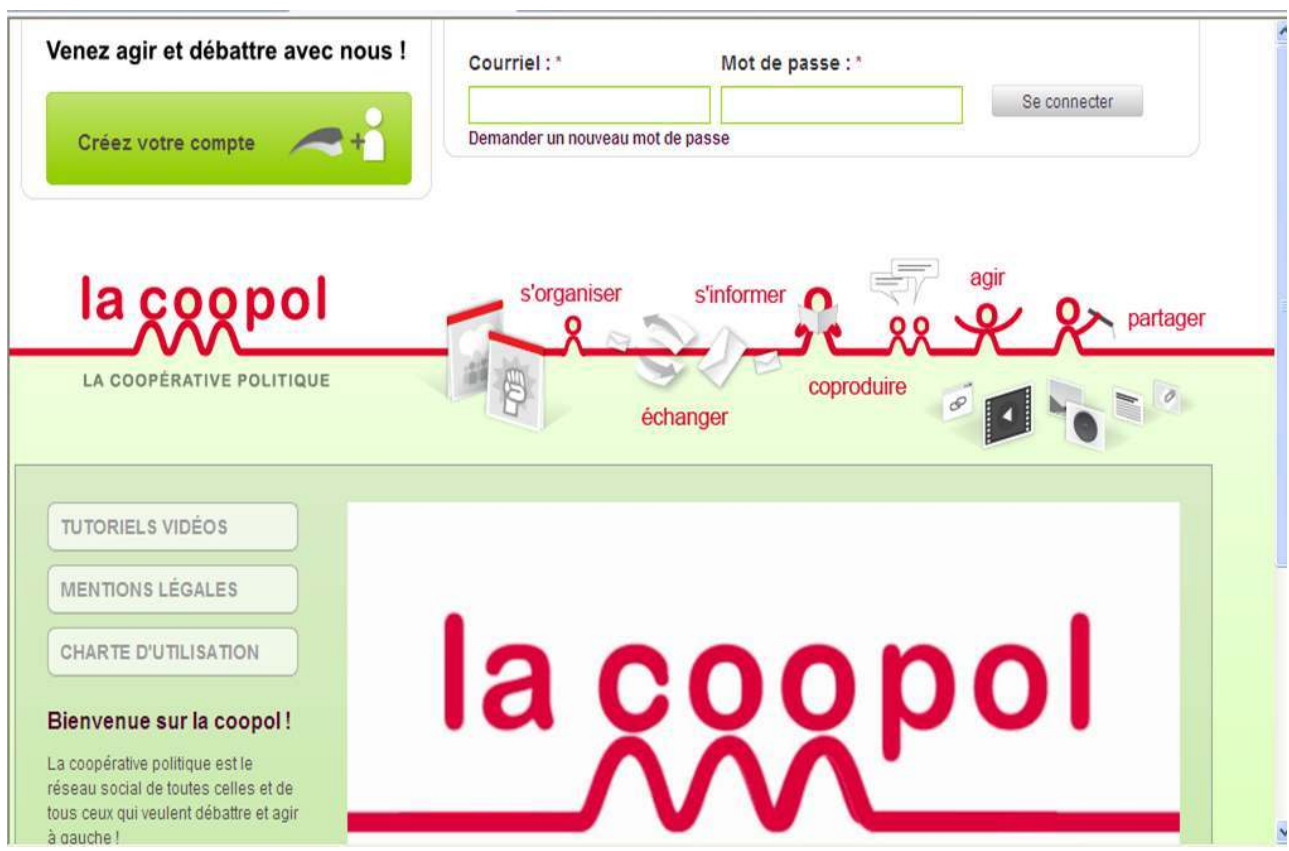

COPIE D'ÉCRAN : http://www.lacoopol.fr, conSULTÉ 2.10.2018)

La conséquence de ces interventions de «lissage», voire de "gommage» de l'affrontement intra-partisan a été quasi immédiate (Oger \& Ollivier-Yaniv, 2006). En recadrant les débats afin de les inscrire dans la culture délibérative propre au PS, les responsables du site ont amené les internautes à délaisser un outil qui ne correspondait pas à leurs attentes. Sur la Toile, le débat est en effet censé être plus libre qu'en section. Or, ce dispositif technique a reproduit en ligne l'architecture hors ligne du PS, sans l'adapter à la culture numérique. Alors que La Coopol avait été conçue au départ pour ouvrir le parti sur l'extérieur, elle a été pour l'essentiel fréquentée par des adhérents ayant des responsabilités au sein du parti et soucieux de mener des actions ou de communiquer sur Internet, organisant ainsi sa propre clôture organisationnelle et donc, à une vitesse cependant moins rapide qu'à l'UMP, la fin de son projet politique et technique initial. Les militants socialistes ont ainsi délaissé la plateforme au profit de Facebook.

«Le contact avec des militants d'autres Fédés, on le fait par Facebook déjà. Tous les jeunes ont déjà un compte Facebook en parallèle. On n'y trouve pas grande utilité [dans la Coopol]. Et les vieux entre guillemets, s'ils n'ont pas de compte Facebook, je ne sais pas s'ils utilisent la Coopol pour autant. J'ai l'impression que ceux qui vont sur la Coopol, c'est ceux qui ont déjà un compte Facebook oui déjà l'habitude de se servir des outils numériques. Et c'est [sic] pas le cas de tous les militants non plus. » 23

La mise en œuvre de ces techniques de neutralisation de ce qui pourrait apparaître comme du "désordre interne " n’a cependant pas été le seul frein au développement d'une parole libre sur le réseau. D'autres éléments tels que l'influence du cadre $\left(\right.$ Marcoccia, 2003) ${ }^{24}$, les spécificités techniques, la légitimité, le sentiment de compétence, etc. ont pu également limiter le contenu des propos tenus par les « coopains».

«Pour analyser la manière dont se déroulent des échanges médiatisés par ordinateur, on doit donc prendre en compte l'influence du dispositif technique luimême. En effet, il joue un double rôle : à la fois cadre (il définit les sujets, par exemple) et instrument (il rend possible la discussion)» (Marcoccia, 2003 :12). 
technique sur l'organisation territoriale du parti. Comme le souligne ce responsable, « La Coopol, c'est une transposition de l'architecture, des hiérarchies partisanes, c'est-à-dire les militants, les sections, les fédérations, les instances nationales. C'est une contrainte qu'on avait de structurer cette plateforme $»^{25}$. A l'image du procédé déjà mis en place lors de la création des militants à " 20 euros », le PS a souhaité que "le virtuel rencontre le réel ». Chaque coopain pouvait ainsi être rattaché, s'il le souhaitait, à une section physique du PS. Le militant virtuel n'était donc pas déterritorialisé, mais bien inscrit dans le découpage géographique de son parti. Conçu pour moderniser la façade institutionnelle du parti, ce dispositif de discussion a proposé un cadre paradoxalement traditionnel pour l'activité des internautes.

Il ne suffit donc pas de posséder les connaissances techniques pour devenir acteur du débat en ligne. Beaucoup d'internautes qui ont franchi le cap de l'inscription à La Coopol sont restés silencieux sur cette plateforme et n'ont fait qu'observer ce qui s'y passait sans jamais intervenir. Pis, les débats organisés ont pu donner l'impression d'être confisqués par quelques membres de La Coopol. Par exemple, le groupe « Pour le droit de vote des étrangers résidents en France aux élections locales » était le plus "peuplé $»^{26} \mathrm{de}$ La Coopol avec ses 2357 membres. Du 16 octobre au 20 juillet 2011, 337 posts (messages) ont été enregistrés au sein de ce groupe, dont 158 sont attribués à la même personne. Le second plus gros contributeur, pendant la période indiquée, a posté 57 messages. Seulement deux contributeurs sur 2357 membres ont donc été à l'origine de 215 messages, soit environ deux tiers du contenu du groupe.

Les promesses de "plus d'horizontalité » avec l'arrivée du web dans l'organisation partisane, dans les discours des responsables web («Nous allons introduire de l'horizontalité dans un système pyramidal $\aleph^{27}$ ) et des dirigeants politiques socialistes, ont créé en fait des déceptions du côté des militants, faute de possibilités réels de contacts avec les cadres du parti. Comme le souligne ce militant, «Internet n'a rien changé. Les cadres sont peu présents (ou distants) sur Internet. C'est seulement un bon moyen pour garder contact avec des militants que l'on connaît déjà. Mais ça, on pouvait le faire sans Internet. ${ }^{28}$ Autrement dit, les militants ont été à la fois déçus en pratique (en lien avec les dispositifs mis en place) et sur le fond (la possibilité même de réformer le système), ce qui a pu alors amplifier les désillusions vis-à-vis du PS et de ses projets de développement d'outils numériques participatifs. Jugée alors trop modérée, au sens numérique et politique du terme, La Coopol a alors été supplantée, d'un côté par Facebook pour les militants en demande de débats et d'échanges, et de l'autre par Twitter pour la direction du PS et par l'équipe de campagne de François Hollande en raison du caractère directif et pleinement assumé de l'outil.

\section{De la difficulté de créer des civic tech à l'intérieur de partis politiques}

Conçues pour remettre à jour la démocratie partisane, les initiatives numériques menées par l'UMP et par le PS ont rapidement montré leurs limites. Pour que les citoyens s'autorisent à s'exprimer librement sur des espaces de discussion partisans, de nombreuses conditions semblent donc nécessaires : une modération tolérante, un cadre peu contraignant, des compétences techniques et culturelles des membres (ou du moins 
un sentiment de compétence ${ }^{29}$ ), un contexte socio-technique spécifique et l'envie enfin de débattre en ligne. En restant dans un entre-deux, ces partis n'ont pas réussi à respecter ces réquisits. En cela, l'UMP et le PS ont souligné par ricochet la difficulté pour des partis de marier dans un même mouvement renouvellement des formes de médiation et travail de domestication des expressions citoyennes. Faute d'avoir su ou pu dépasser les tensions qui pouvaient exister entre cette injonction à la prise de parole libre et la nécessité de contrôler les expressions internes, ces plateformes partisanes à visée communautaire ont alors buté sur la spécificité culturelle des organisations dont elles dépendaient organiquement et financièrement. Cette volonté de cloisonnement semble s'effriter de plus en plus avec l'arrivée de «nouveaux " partis politiques qui bousculent les façons de faire traditionnelles. Il est alors intéressant de porter le regard sur le cas espagnol et l'usage du numérique que propose Podemos avec par exemple la mise en place d'un Programme Participatif, discuté ensuite par les citoyens sur les réseaux sociaux (Petithomme, 2017).

Cette tension n'est pas pourtant propre aux "vieux" partis. Les logiques d'accompagnement développées par l'UMP et le PS se font également jour dans d'autres secteurs, plus particulièrement marqués par les modèles entrepreneuriaux. En raison du rôle institutionnellement confié aux formations politiques, elle est cependant d'une autre nature. Comme le souligne Clément Mabi, « la question de l'équipement ne doit pas faire passer au second plan la réflexion sur la nature du projet politique embarqué par ces dispositifs » (Mabi, 2017).

L'échec de ces civic tech à visée partisane au sein de l'UMP et du PS montre ainsi que les formations politiques de gouvernement ne se détachent pas de leur mode d'organisation. Les frontières partisanes sont certes assouplies, mais en aucun cas abattues. La conversion des partis à l'Internet est donc tout sauf neutre. Pour une raison simple : cette conversion doit en grande partie son succès au fait qu'Internet a été considéré dès le départ comme une technique de gouvernement dont les dirigeants des partis ont usé des fonctionnalités en fonction d'objectifs internes (rationaliser le militantisme de terrain) et d'affichage externe (ouvrir et prendre en compte la parole citoyenne, sans que cela soit réellement le cas).

Même si ces deux cas d'étude - Les Créateurs des possibles et La Coopol - apparaissent en premier lieu comme des échecs dans une visée de participation citoyenne, voire d' empowerment, ils peuvent aussi être appréhendés comme une étape sur le long chemin de l'amélioration de la démocratie partisane et plus largement citoyenne, même si en suivant la typologie proposée par Clément Mabi, il est difficile de considérer que ces initiatives numériques à visée participative ont fait de leurs développeurs des « hackeurs embarqués ", au sens où des entrepreneurs du changement auraient réussi, malgré des freins internes importants, à réformer le système de l'intérieur.

Ces premiers discours de participation citoyenne se sont majoritairement reconfigurés en enjeux de mobilisation. L'engagement citoyen en ligne, bien qu'affiché comme prioritaire, n'est pas (ou plus) un axe prépondérant pour ces deux partis politiques qui ont surtout vu le numérique comme un outil de rationalisation du militantisme de terrain, voire comme un pur "effet de rustine» (Mabi, 2017). Ce processus d'institutionnalisation n'est cependant pas lié à la spécificité des projets portés par ces deux plateformes. Déjà en 2008, la dimension participative avait été repensée dans l'action. Comme le souligne cette responsable numérique, «le participatif de Désirs d'Avenir, c'était un participatif sur le contenu, quasiment sur le contenu programmatique. Là, ce n'est pas du tout ça. Là, on 
était dans du participatif, comme je l'appelle, de mise en bouche. On n'était pas dans la consultation. Le projet, le programme, c'étaient ceux du candidat. Là où il y a eu du participatif c'était sur un plan très global. C'était de ne pas s'adresser aux gens de façon très passive comme pur récepteur de message, mais s'adresser à eux en tant qu'acteurs de la campagne et en leur donnant des modalités d'action, en leur proposant vraiment d'agir. $\aleph^{30}$ Dans ce cadre, la participation, entendue comme mobilisation, s'inscrit de plus en plus dans un flottement opportuniste du terme, faisant ainsi écho aux controverses scientifiques sur la plasticité du concept de participation, avec notamment les travaux de Eulàlia Puig-i-Abril et Hernando Rojas (2009) sur la notion de "participation politique expressive ». La tendance est désormais au travail marketing de l'idéal d'empowerment porté par les civic tech. Autrement dit, accroitre le pouvoir citoyen apparait davantage aujourd'hui comme un argument de vente pour de nouvelles start-up spécialisées en stratégie électorale (Theviot, 2016b) ou une activité monétisée par des firmes que comme un dessein à visée démocratique (Cardon et Casilli, 2015), confortant ainsi le marché déjà fortement développé des civic tech entrepreneuriales et la légitimation de nouvelles pratiques de ciblage des électeurs par les data ou la gestion des données numériques.

\section{BIBLIOGRAPHIE}

BACQUÉ Marie-Hélène \& $\mathrm{B}_{\text {iewener }}$ Carole (2015). L'empowerment, une pratique émancipatrice, Paris, La découverte/Poche.

BADOUARD Romain \& MABI Clément (2015). «Le débat public à l'épreuve des controverses », Hermès. La Revue, 1 (71), pp. 145-151.

BARBONI Thierry \& TREILLE Eric (2010). «L'engagement 2.0. Les nouveaux liens militants au sein de l'e-parti socialiste », Revue française de science politique, 60(6), pp. 1137-1157.

BLONDIAUX Loïc (2011). « Métamorphise de la démocratie : vers une démocratie réelle », Conférence donnée au cours de la session 2011 des Semaines sociales, « La démocratie une idée neuve ».

BREINDL Yana \& FRANCQ Pascal (2008). « Can Web 2.0 Applications Save e-Democracy? A Study of How New Internet Applications May Enhance Citizen Participation in the Political Process Online ", International Journal of Electronic Democracy, 1, pp. 14-31.

CARDON Dominique (2010). La démocratie Internet, Paris, La République des Idées.

CARDON Dominique \& CASILLI Antonio (2015). Qu'est-ce que le Digital Labor, Paris, INA éditions.

CHADWICK Andrew (2007). « Digital Network Repertoires and Organizational Hybridity », Political Communication, 24, pp. 283-301.

Снамват Pierre (2000). «La démocratie assistée par ordinateur », Cahiers politiques, 4, pp. 47-75.

COLEMAN Stephen (2005). « New mediation and direct representation: reconceptualizing

representation in the digital age ", New Media \& Society, 7(2), p. 177-198. 
Collin Philippa (2015). Young Citizens and Political Participation in a Digital Society. Addressing the Democratic Disconnect, London: Palgrave.

DAlton Russel J. \& WATtenberg Martin P. (dir.) (2000). Parties Without Partisans. Political Changes in Advanced Industrialized Democracies, Oxford: Oxford University Press.

DATCHARY Caroline (dir.) (2012). La dispersion au travail, Toulouse, Octares.

DESQUINABO Nicolas (2008). « Dynamiques et impacts des propositions politiques dans les webforums partisans », Réseaux, 26(150), pp. 107-132.

GIBSON Rachel K. (2012). « From Brochureware to 'MyBo': An Overview of Online Elections and Campaigning », Politics, 32(2), pp. 77-84.

GOURGUES Guillaume (2012). «Les fonctionnaires participatifs : les routines d'une innovation institutionnelle sans fin(s) », Socio-logos. Revue de l'association française de sociologie, 7 [En ligne], mis en ligne 27.03.2012, consulté 07.04.2018. URL : http://journals.openedition.org/socio$\operatorname{logos} / 2654$.

LEES MARSHMENT Jennifer (2011). Routledge Handbook of Political Marketing, Londres, Routledge.

HAEGEL Florence (2007). « Le pluralisme à l'UMP. Structuration idéologique et compétition interne ", in Haegel Florence (dir.), Partis politiques et système partisan en France, Paris, Les Presses de Science Po.

LEFÉBURE Pierre \& $\mathrm{S}_{\text {ÉCAIL }}$ Claire (dir.) (2016). Le Défi Charlie. Les médias à l'épreuve des attentats, Paris: Lemieux.

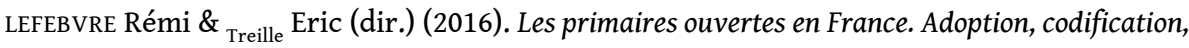
mobilisation, Rennes, Presses universitaires de Rennes.

MARCoccIA Michel (2003). «Parler politique dans un forum de discussion», Langage et société, 104, pp. 9-55.

MABI Clément (2017). «Le débat CNDP et ses publics à l'épreuve du numérique. Entre espoirs d'inclusion et contournement de la critique sociale », Cahiers COSTECH, 1.

MERMET Laurent (2007). «Epilogue. Débattre sans savoir pourquoi : la polychrésie du débat public appelle le pluralisme théorique de la part des chercheurs », in Revel Maurice, Blatrix Cécile., Blondiaux Loïc Fourniau Jean-Michel, Hériard Dubreuil Bertrand et Lefebvre Rémi, Le débat public : une expérience française de démocratie participative, Paris, La Découverte.

MONNOYER_SMITH Laurence (2011). Communication et délibération. Enjeux technologique et mutations citoyennes, Paris, Lavoisier-Hermes.

OGER Claire et ollivier-Yaniv Caroline (2006). « Conjurer le désordre discursif. Les procédées de « lissage » dans la fabrication du discours institutionnel », Mots. Les langages du politique, 81, pp. 63-77.

PÈNE Clémence (2013). « La nouvelle science électorale américaine ", Revue politique étrangère, 2, pp. 127-139.

Petithomme Matthieu (2017). «L'« activisme institutionnel » de Podemos : entre contestation et transformation organisationnelle », Variations, 20 [En ligne], mise en ligne 25.04.2017, consulté 07.04.2018. URL : http://journals.openedition.org/variations/833.

PUIG-I-ABRIL Eulàlia \& ${ }_{\text {Rojas }}$ Hernando (2009). « Mobilizers Mobilized: Information, Expression, Mobilization and Participation in the Digital Age ", Journal of Computer-Mediated Communication, 14, pp. 902-927. 


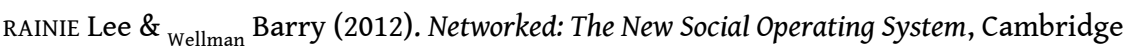
Massachusetts, MIT Press.

RoSANVAllon Pierre (2006). La contre-démocratie, la politique à l'âge de la défiance, Paris, Seuil. SCARROW Susan (2014). Beyond Party Members, Oxford, Oxford University Press.

STROMER-GALLEY Jennifer (2014). Presidential Campaigning in the Internet Age, Oxford, Oxford University Press.

TALPIN Julien (2016). Community Organizing. De l'émeute à l'alliance des classes populaires aux EtatsUnis, Paris, Raisons d'agir.

THEVIOT Anaïs (2018). Faire campagne sur Internet, Lille, Presses Universitaires de Septentrion.

THEVIOT Anaïs (2016a). « Les primaires : un terrain d'expérimentation de l'innovation politique ? Le cas de la campagne d'A Juppé en 2016. Une mobilisation scientifique orchestrée par les data ", in Lefebvre Rémi et Treille Eric (dir), Les primaires ouvertes en France. Adoption, codification, mobilisation, Rennes, Presses universitaires de Rennes.

THEVIOT Anaïs (2016b). « Les data : nouveau trésor des partis politiques ? Croyances, constitutions et usages comparés des données numériques au Parti Socialiste et à l'Union pour un Mouvement Populaire ", Politiques de communication, 6, pp. 137-166.

VEDEL Thierry \& Ward Steven (2006). « Introduction. The Potential of The Internet Revisited », Parliamentary Affairs, 2, pp. 210-225.

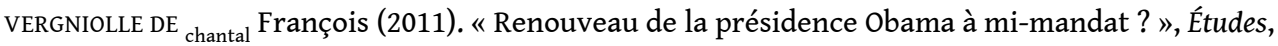
4(414), pp.439-450.

WRIGHT Scott (2012a). « From "third place" to "third space". Everyday Political Talk in Online Non-Political Spaces », Javnost-The Public, 3, pp. 5-20.

WRIGHT Scott (2012b). « Politics as usual? Revolution, normalization and a new agenda for online deliberation », New Media \& Society, 14(2), pp. 244-261.

\section{NOTES}

1. Fondé en 2009 à Los Angeles par J. Gilliam, NationBuilder se décrit comme un "système d'exploitation de communauté ». Utilisé lors de la campagne de B. Obama en 2012, il a été employé aussi bien par le Labor Party australien que par Amnesty International, AirBnb ou Handicap International.

2. Présentation des Créateurs de possibles, http://www.lescreateursdepossibles.com (consulté le 12 mars 2010 - il n'est désormais plus accessible).

3. Martin, adhérent PS de la Fédération de Gironde. Entretien du 25 octobre 2011.

4. «Coopol et créateurs de possibles, Epicentre pour le nouveau centre, Les démocrates pour le Modem, Europe Ecologie qui va lancer sa nouvelle version... Nouveau sujet à la mode : tout le monde veut son mybarackobama.com ", Meilcour.fr, blog politique, http://www.meilcour.fr/ polito/rseaux-sociaux-politiques-quelques-remarques.html (consulté le 26 novembre 2013).

5. Baptiste Roynette, directeur du web à l'UMP. Entretien du 31 janvier 2013.

6. Pierre Chassat, directeur de la communication de l'UMP. Entretien du $1^{\mathrm{er}}$ mars 2012.

7. «L'UMP n'a pas, contrairement au PS, la culture de cette démocratie interne », Libération, Interview de Florence Haegel, 20 novembre 2012, http://www.liberation.fr/ 
politiques/2012/11/20/1-ump-n-a-pas-contrairement-au-ps-la-culture-de-cette-democratieinterne_861650 (consulté le 26 novembre 2013).

8. Benoît Thieulin, directeur de la Netscouade et directeur web de la campagne de Ségolène Royal pour l'élection présidentielle de 2007. Entretien du 29 février 2012.

9. Demander aux citoyens leurs idées pour un parti au pouvoir peut laisser penser que le gouvernement en manque. Cet argument a été utilisé par les partis concurrents afin de critiquer l'UMP.

10. Ces sites avaient compris que le moteur de la mobilisation réside dans ces mécanismes de médiatisation auprès de pairs, de rencontres, de chaleur humaine, de liens faibles créés par les messages, d'émulation des scores, etc.

11. Emile Josselin, directeur adjoint à la direction du web à Solférino et directeur de la campagne numérique de M. Aubry pour les primaires socialistes en 2011. Entretien du 27 janvier 2012.

12. Le président des JP, chargé de la promotion des Créateurs de possibles en 2010, a certifié lors d'un entretien ne pas connaître cette plateforme. Suite à nos relances, cet enquêté a demandé à arrêter l'entretien pour se rendre aux toilettes. A son retour, il a préféré aborder un autre sujet.

13. Nous pensons par exemple à Pierre Chassat (pro-Copé), directeur de la communication de l'UMP au siège du parti, arrivé sous la gourvernance de Jean-François Copé.

14. La création de ce réseau a été fortement médiatisée puisqu'il a été dévoilé lors des vœux de Martine Aubry, Première secrétaire du PS. L'enjeu était alors de donner une image de modernité du parti qui sait se saisir des innovations technologiques.

15. Message reçu lors de l'inscription à la Coopol.

16. Libération, 29 décembre 2009.

17. Message d'accueil sur le réseau partisan, la Coopol. http://www.laCoopol.fr/ (consulté le 28 septembre 2013).

18. « Coopol, pour se faire plein de coopains au PS », France2.fr, 8 février 2010.

19. Message reçu lors de l'inscription à la Coopol.

20. Sont ainsi appelés les membres inscrits de la Coopol. Il s'agit d'un jeu de mot, né de l'association des termes « copains » et « Coopol».

21. Matthieu, responsable des communautés web au PS. Entretien du 10 février 2011.

22. D'autant plus qu'avait été lancé, une semaine plus tôt, le réseau social de l'UMP : les comparaisons étaient faciles pour les médias.

23. Juliette, militante au PS et animatrice fédérale MJS de Gironde. Entretien du 1er juin 2011.

24. «Pour analyser la manière dont se déroulent des échanges médiatisés par ordinateur, on doit donc prendre en compte l'influence du dispositif technique lui-même. En effet, il joue un double rôle: à la fois cadre (il définit les sujets, par exemple) et instrument (il rend possible la discussion). » (Marcoccia, $2003: 12$ ).

25. Rémi, chargé de mission responsable de la Coopol à la Netscouade. Entretien du 27 janvier 2012.

26. Nous reprenons ici le terme employé sur la Coopol pour classer les quinze premiers groupes possédant le plus de membres.

27. Valério Motta, lors du lancement de la Coopol ; cité dans l'article du journal Le Figaro, intitulé «L'UMP et le PS se mettent à l'heure des réseaux sociaux », paru le 10 novembre 2009.

28. Louis (anonymisé), 52 ans, adhérent au PS, Fédération de Paris. Entretien du 9 juin 2012.

29. Le sentiment de compétence constitue généralement une traduction subjective de la position sociale.

30. Claire, responsable du porte-à-porte, puis coordinatrice de l'équipe numérique de $\mathrm{F}$. Hollande. Entretien du 4 juin 2012. 


\section{RÉSUMÉS}

En créant Les Créateurs du possible et La Coopol, l'UMP et le PS ont souhaité s'inspirer de l'émergence du mouvement des «civic tech" pour faire remonter les idées citoyennes et pour ouvrir les débats partisans aux non-adhérents. Toutefois, ces ambitions se sont vues fortement contrariées par le manque de participation en ligne et la volonté d'encadrer "par le haut» le fonctionnement de ces plateformes d'inclusion militante.

By creating The Creators of the Possible and La Coopol, the UMP and the PS wanted to draw inspiration from the emergence of the « civic tech» movement to bring up citizen ideas and to open partisan debates to non-members. However, these ambitions were strongly thwarted by the lack of online participation and the desire to frame "from above» the functioning of these activist inclusion platforms.

\section{INDEX}

Keywords : PS, UMP, political communication, civic tech, participatory platforms, online participation

Mots-clés : PS, UMP, communication politique, civic tech, plateformes participatives, participation en ligne

\section{AUTEURS}

\section{ANAIIS THEVIOT}

Université catholique de l'Ouest, Arènes

\section{ÉRIC TREILLE}

Université de Rennes, Arènes 